\title{
Function analysis Process Based on the Components Unit Xiaowen $\mathrm{Chi}^{\mathrm{a}}$, Changqing $\mathrm{Gao}^{\mathrm{b}^{*}}$, Pengjie Zhai ${ }^{\mathrm{c}}$ and Yuansong Zheng ${ }^{\mathrm{d}}$ \\ School of Mechanical Engineering, University of Jinan, Jinan 250022, P.R. China \\ a18764117726@163.com, ${ }^{\text {b* }}$ me_gaocq@ujn.edu.cn, ${ }^{c}$ zhaipj2012@163.com, d1248995968@qq.com
}

Keywords: Component Unit, Graph Theory, Function Analysis, Adverse Effects, ENV Model, Function Chain.

Abstract. The functional information model of the technical system based on the component unit model is the basis of the analysis of computer aided function. Through the introduction of graph theory knowledge, establishing technical system function directed graph's adjacency matrix and reachable matrix. The establishment of technical system of multilevel advanced directed graphs can directly determine the technology system of internal parallel chain. Using the ENV model to analyze the causal relationship between the adverse effects in the chain, and to find the key adverse effect of solve the problem.

\section{Introduction}

Functional analysis is the most important part of product innovation process. The existing functional analysis methods are mostly drawing a rough representation on the drawing by designers based on their background knowledge and understanding, no discussing the relationship between components, the analysis process is complicated and the analysis results with random. There is not analysis the adverse effect relationship for the condition of the effect chain has multiple adverse effects, designers face the complex adverse effects chain are unable to start. Therefore, it urgent requests a set of mature functional analysis method in product innovation design.

The study through the study on graph theory, constructing the model based on component units functional analysis, analyzing adverse effects chain, finding a process that can be achieved for functional analysis method based on the unit, which made preparation for extracting relevant means in knowledge space.

\section{Information Modeling of Technical Systems Based on Component Units}

Functional analysis is a technical system functional modeling process. The function information model which is in the interaction between components as the main content, it is the structured expression for technical system general function achieving principle level. This expression can be more directly reflect the function implementation principle of product [1].

Interaction between the two components forms a component unit. You can establish the function of the product system model by several components unit combining a role relationship, in order to i mplement structural representation of the system functions as a whole. Several components interact to achieve the function of technology systems.

The component unit model is basic to describe technology system functional model, which reflect $\mathrm{s}$ the effect relationship between components. Using the knowledge of graph theory to indicate the ef fect relationship between components of technology system in the form of a matrix, not only can be tter record complex relationships between components, but also conducive to realize to write the cha in of the technology systems through computer programming language, which make technical syste $\mathrm{m}$ function analysis faster and more accurately.

\section{Function chain structure model of the technology system based on reachable matrix}

By using the theory and method of artificial intelligence, developing the Element-Name-Value model of object-oriented, referred to as ENV model [2-4].ENV theory thinks that the first is the description 
of the system and the specification of the problem before solving problem. Description of any problems must be focused on the problem causes, clearly define the main elements, elements of the evaluation parameters and its corresponding value, as well as the elements of the problem and the corresponding parameters and values. ${ }^{[3]}$ As shown in Figure 1.

In the ENV model, two classes of parameters are constructed by OTSM: a class called evaluation parameter, another class is called the control parameter. It has a pair of opposite values. When the value is taken, the positive and negative effects are produced on the two evaluation parameters. The control parameters are the key parameters to solve the problem of technological innovation. [5]

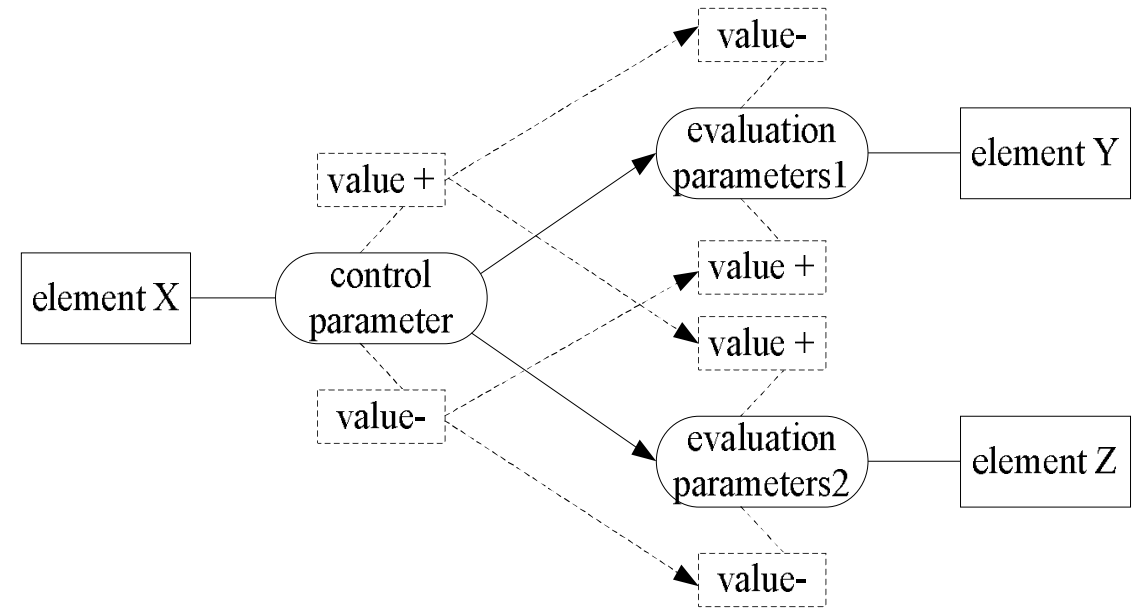

Figure $1 \mathrm{ENV}$ model

As the different function of the system and the components of the role are in a variety of ways, so the structure of the functional directed graph is varied. Through functional information modeling to get the function of the structure of directed graph is also diverse. In technical systems, the generation of a bad effect is often associated with the properties of the components and the properties of the components. According to the ENV model, we can analyze the attributes of negative effect components. Through the analysis of related attributes, we can find out causal relationship between adverse effects and effect relationship, but also through the analysis to find a new way of thinking and solutions to achieve product innovation. There is a causal relationship between multiple adverse effects in a technical system of directed graph. The occurrence of adverse effects may cause the occurrence of another or more adverse effects, or the occurrence of an adverse effect is caused by the occurrence of one or more adverse effects. In order to solve the problem of the convenience, through the reachable matrix, we sum up four kinds structure of technical system: Series Type, Parallel Type, Mixed Type, Feedback Type. And use the diagram to express. The model of chain structure is shown in Table1.

Table1 model of chain structure

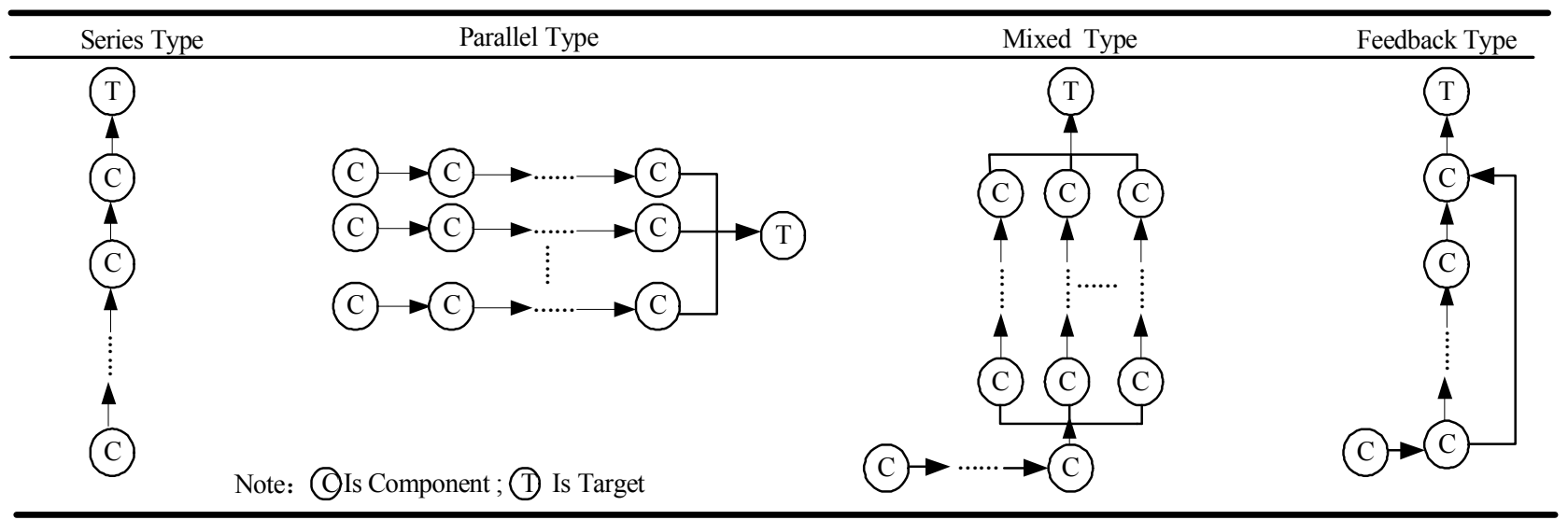

In the form, causal relationship may exist between the adverse effects of relationship, adverse effects 1 may lead to adverse effects 2 , adverse effects 2 may lead to adverse effect 3 , adverse effect 3 had an effect on the adverse effect of 1 , forming a circular negative effect chain with feedback effect. 
Through the structure of the chain, it can be seen the complexity of the relationship between the technical system components. In the elimination of adverse effects in the process, change one or a few of the component unit structure or properties, the introduction of resources and functional unit binding, cutting and other behavior, may have some effects on the other components or technology system. So in the actual problem solving process, should be as far as possible to shorten the length of the chain length, to find out the cause and effect relationship, find out the root cause of adverse effects, and optimize the adverse effects, in this way can help eliminate the adverse effects.

Function and action are the products of the attributes between the two components.[6]Using the ENV model, the analysis of the ENV model from the most advanced components of the adverse effect, and then the two components of the analysis were analyzed.

Component analysis termination rule:

(1)At least one of the two components is the lowest level components.

(2)Components of the two components through the ENV model analysis is the component itself.

\section{Analysis scheme of different structural forms}

The function relationship of the internal components of the chain is more complex, and the order of the adverse effects is needed. First, choose the most critical adverse effects solve to reduce the difficulty of solving. According to the actual problem, the adverse effect of the actual problem is re constructed, and the complex adverse effect chain is divided into several simple forms, which are integrated to get the key of adverse effects.

Method one: Trace the ultimate cause.

The ultimate reason is the key reason that causes the bad effect of the technical system. Tracing the ultimate reason is a kind of method for the structural treatment of the root cause of the bad effect. The ultimate cause of the problem is a great significance for e eliminating the negative effect of the components and the root cause of the conflict. Through the ENV model analysis, the components of the highest points are generally "the ultimate cause".

Method two: The ultimate reason ranking.

There may be more than one "ultimate reason" of the technical system, which is the most likely to occur in the structure mode of parallel type and mixed type. For more than one ultimate reason we do not know which one to choose solute is good, then we need to make a number of final reasons for the importance of sorting, selection of the most critical components to solve the adverse effects.

Take different methods to solve the problem according to the characteristics of the four kinds of technology system of chain structure model.

(1) Series Type: Apply method 1 to determine the adverse effect of 1 for the "ultimate reason", which need to be resolved.

(2)Parallel Type: In the Parallel Type chain structure model, first, you need to decompose parallel multiple role in the chain into a plurality of parallel Series Type role in the chain, then each a series of chain were analyzed. Then according to the solving method of Series type chain structure model to solve. To resolve the adverse effect of Parallel Type of chain structure model of the "ultimate reason" may have multiple, then according to the second method for ranking and choosing the key to solve the adverse effects.

(3)Mixed Type: In the Mixed Type, exist simultaneously in Parallel Type and Series Type, first of all, the parallel chain is decomposed into a series of parallel chains, and then the key is obtained by using the method 1 to analyze" ultimate reason"

(4) Feedback Type: If the feedback effect chain model existing in the Parallel Type, first of all decompose parallel chain into a simple Series Type chain model, reuse method 1to analyze "ultimate reason" to seek the key to adverse effects.

In the practical problem analysis, two methods are needed.

Solving process is shown in the following Figure2: 


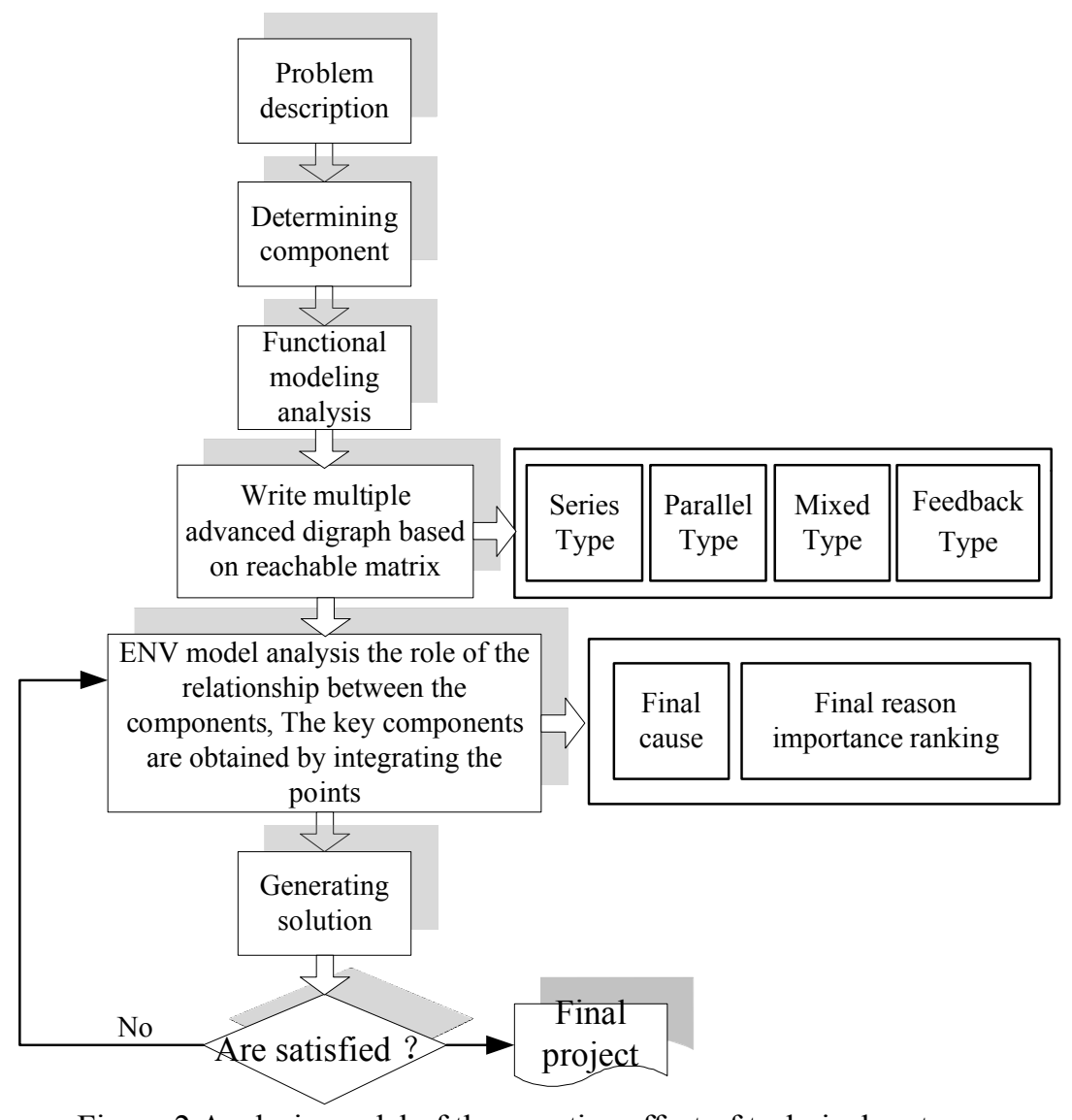

Figure 2 Analysis model of the negative effect of technical system

\section{Improvement design of thin wall material folding equipment}

Disposable gloves, shower cap or disposable foot sets and irregular shape of the thin-wall lightweight materials, in the hotel are used more and more widely. But at the moment, the folding packaging technology is relatively backward, basically rely on manual folding or a mechanical device folded into a certain shape to reduce the volume and transmitted to the next packaging, which waste a lot of manpower and material resources.

As shown in Figure 3, it is an existing device for folding the thin wall material.

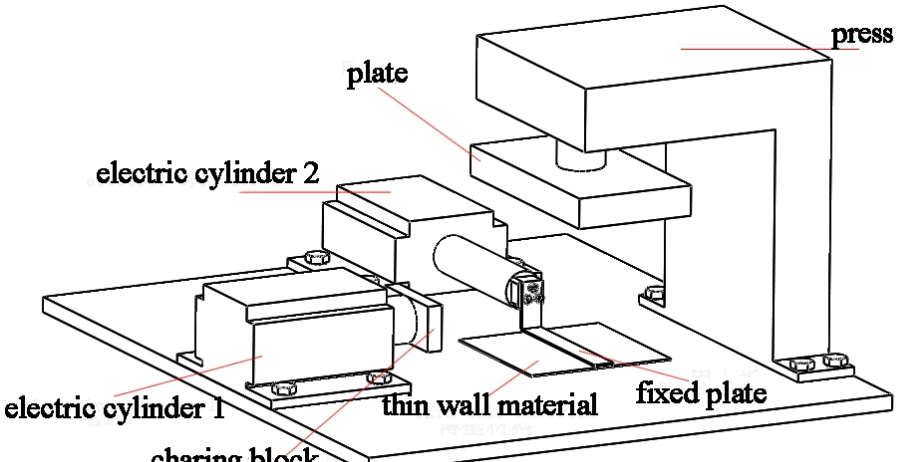

charing block

Figure 3 Folding device of thin material

Below we follow the technical system of the negative role of the chain analysis process to the system analysis, the process is as follows:

The first step: Problem description.

The method of folding thin-wall materials, which is through electrostatic interaction, through the charging of thin material, make thin wall material with certain charge, charged block zone and thin wall materials instead of charge, through different charge attraction of thin-wall materials are folded, by repeatedly charging folded to form multilevel folding effect.

The second step: Determine the components. 
1. electric cylinder $1 ; 2$.electric cylinder $2 ; 3$.charged block; 4.the press; 5 .fixed-plate; 6. plate; 7. thin-wall material.

As shown in Table 2.

Table2 Modeling and analysis of the function of thin wall materials

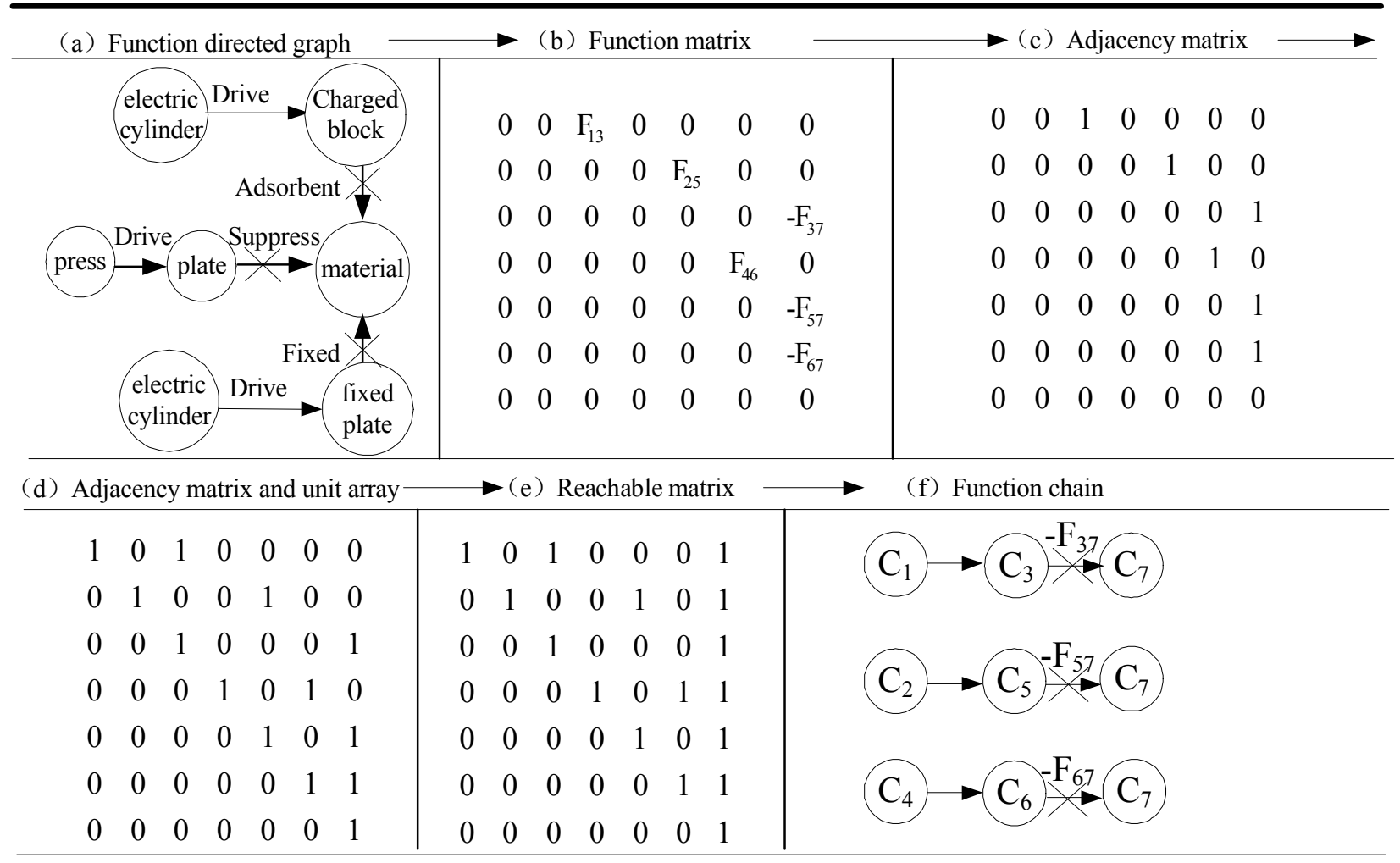

The fourth step: Write advanced multilevel directed graph based on reachable matrix.

Advanced multilevel directed graph is shown in Figure 4.

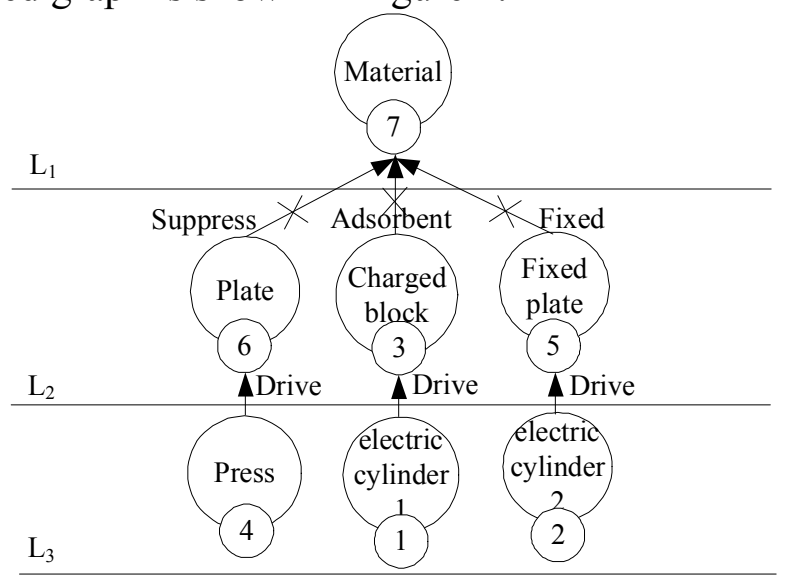

Figure 4 multilevel advanced directed graph of thin wall material folding device

Through the multilevel advanced directed graph can be seen intuitively, chain structure of the thin-wall material folding equipment is Parallel Type.

The fifth step: By ENV model analysis to the key to solve the negative role of components.

According to the method of the parallel structure model of the chain structure, the parallel multiple function chains are decomposed into a series of parallel Series Type, And then analyze each of the Series Type chain.

According to the advanced multilevel directed graph, the Series Type chain of the technical system is as follows:
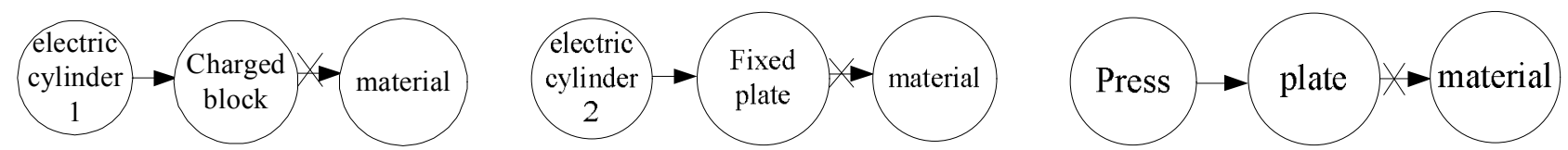
After the ENV model analysis, the causal relationship between the following components is obtained.

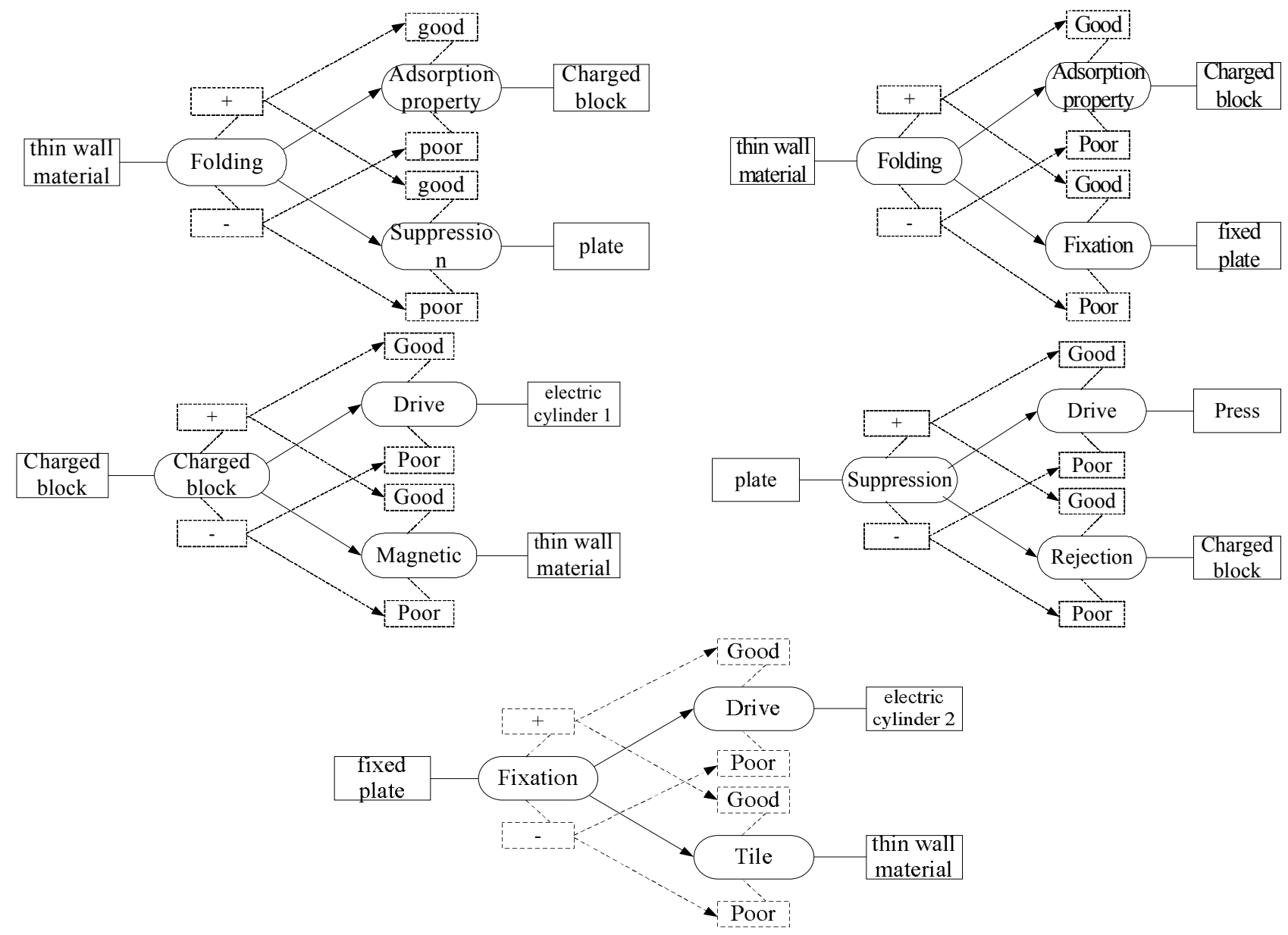

The sixth step: solution

To sum up, the correlation integral between components is shown in Figure 5.

\begin{tabular}{|c|c|c|}
\hline $\begin{array}{c}\text { Component } \\
\text { number }\end{array}$ & Component name & Integral \\
\hline 1 & electric cylinder 1 & 1 \\
\hline 2 & electric cylinder 2 & 1 \\
\hline 3 & Charged block & 3 \\
\hline 4 & Press & 1 \\
\hline 5 & fixed plate & 1 \\
\hline 6 & Plate & 1 \\
\hline 7 & thin wall material & 2 \\
\hline
\end{tabular}

Figure 5 Component correlation integral

Through the ENV model analysis of the relationship between the components, we can see that there is a causal relationship between the components of the technology system. According to the above figure, we can see charged block correlation degree is the highest. So, one of the "ultimate reasons" is the charged block.

Analysis of the shortcomings of the technology system:

(1)The thin-wall material and the charged block in the process of folding need to add charge many times, the operation is complicated and the efficiency is low.

(2)During the operation of the folding device, it is required to participate. Through the human respectively, the thin-wall material and the charged mass charge

(3)The stationary plate is driven by the electric cylinder 2 and can be carried forward and backward. When the thin-wall material is folded, the fixed plate is pressed against the thin-wall material. Each fold, the fixed plate needs to be back, the pressing plate presses down on the thin-wall material by the press. Operation process is complicated, repeat. 
(4) High cost of press.

By the analysis of the ENV model and the shortcomings of the causal relationship between the components and technology system can see the method of the static adsorption of the thin-wall material is not the best method.

Generation of solution:

After analysis, the optimization of the engineering parameters is the shape, and the deterioration of the engineering parameters is the time loss. Through the search of conflict matrix, find the invention principle is 14 curved surface, 10 pre-operation, 34 abandoned and recovery, 17 Change dimension.

So, in the design of this improvement, we use the principle of the invention 14curved surface.

(1) The use of multiple wound into a cylindrical shape, into a curved surface, and then to suppress the effect of multilevel folding. According to the function code table, find the corresponding scientific effect, according to the principle of gas pressure to achieve the thin wall material.

Design grasp pump, drilling pump, pump cavity followed by rotary joint, a three-way pipe and valve and positive pressure air and negative pressure air source connection. One end is fixed on the spindle, second one-way pore forming an inward and outward air blowing holes first check. Inhale and crawls through the pump rotation, the thin material good adsorption in an adsorption. As shown in Figure 6.

(2)To suppress the thin-wall material of the winding, the first need to be a good fixation of the cylindrical thin-wall material, and then press the pressing plate.

For a very good fixation of the cylindrical thin-wall material, we design a cylindrical manipulator, which can be coated with a thin-wall material. The manipulator includes a pair of symmetrically arranged occlusion body and drives the shading bodies for closure and separation of the driving mechanism, the occlusion body with a circular cavity of is composed of a plurality of flexible rubber strip. As shown in Figure 7.

(3)For human involvement, we can use the method of cutting of component element type operation. Man's role is to transfer thin-wall material, by adding new components to replace the role of the human. We use the transfer function to achieve the transfer.

The grab pump rotates thin-wall material through the air flowing. We can use the electric spindle drive grab pump. In order to make grabbing pump can from winding station to move to the pressing position, the pendulum rod and the cylinder of the two components to achieve the effect; Pendulum fixed on the machine frame, drive pendulum swing rod of a cylinder is placed between the rod and the rack, by cylinder telescopic action driven pendulum rod swing angle and swing of the two extreme positions respectively in winding and pressing the two work.

Through the above assumptions, the picture to describe the work of winding thin-wall materials is shown as figure 8 .

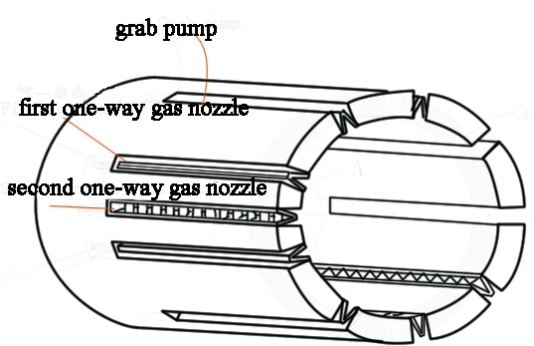

Figure 6 grab pump detail

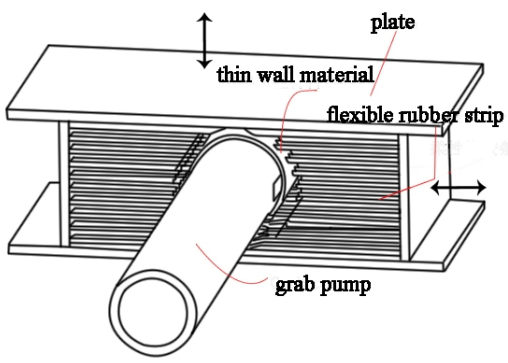

Figure 7 mechanical hand

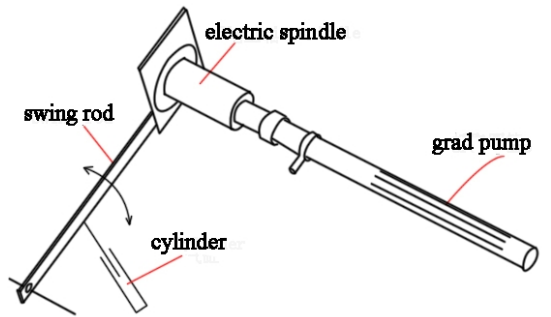

Figure 8 grab pump

Improving of technology design of the system as follows: The thin-wall material tile folded on the convey belt, one end of the grasping cylinder that has only the first one way air hole can breathe in placed upon the thin-wall material and gradually close to the folding wall material. When the end of the folding thin-wall material is adsorbed on the grab pump, the pump rotation and thin-wall material will be folded to the pump. The wound to be folded thin-wall material grab pump transferred to another job position, the working position is provided with a press plate arranged in parallel. The pump by the stomata which can only reverse pulse blowing by the second one-way air hole, making until the folding thin-wall material and grab generates a gap, enables separate, then pump stop blowing out and the two parallel plates press the cylindrical thin-wall material into sheet. Through 
this principle, the effect of multilevel folding is achieved by extrusion. [7]

Using this method also can better realize multilevel folding of the thin-wall material, and the system is simple. It can easy to operate, can improve the work efficiency, can reduce the cost of equipment.

\section{Conclusions}

The conflict is the external manifestation of the bad effects of the technology system, and the essence of the conflict resolution is to eliminate the bad effect of the components. Based on the unit of function analysis model and graph theory knowledge, the establishment of technology system function matrix, adjacency matrix, reachable matrix and function have directed graph can be visualized in the expression of the relationship between each of the components of the system. According to the reachable matrix is proposed technology system function is to several structures of graphs and ENV model of interaction between the component analysis, using different strategies for parallel solution according to the obtained several issues need to be resolved are critical components of the adverse effects, and using the TRIZ to solve adverse effects and satisfactory design scheme is finally obtained. The method is verified by the engineering examples, and the method is proved to be practical.

\section{Acknowledgement}

This work was financially supported by Science and Technology Development Plan Project of Shandong Province (2014GGX106003), Large Equipment Upgrade Project of Shandong Province (2012SJGZ15) and Innovation Method Project (2013IM022300).

\section{Reference}

[1] Changqing Gao, Bo Yang, Jie Lv, Runhua Tan.Conflict elimination method based on component element model[J].Mechanical design,2014,31(7). (IN Chinese)

[2] Cavallucci D,Khomenko N.From TRIZ to OTSM-TRIZ;addressing complexity challenges in inventive design[J].International Journal of Product Development,2007,4(1/2) :4-21.

[3] Cavallucci D.Towards inventive design through management of contraditions[C]//2005 CIRP International Design seminar,Shanghai,2005:271-280.

[4] Khomenko N, Guio R D,Cavallucci D, et al. Enhancing ECN's abilities to address inventive strategies Using OTSM-TRIZ[J].International Journal of Collaborative Engineering,2009,1(1/2):98-113.

[5] Hongchao Yang, Jianhui Zhang, Jinbao Liang, et al.[J].Machine Design, 2014, 4 (31), A product innovation design process model for multi-conflict network. (IN Chinese)

[6] Wucheng Zhang, Min Zhao, Jin Chen, Wei Yao. U-TRIZ based SAFC analysis model [J]. Technology Economy, 2014,12 (33). (IN Chinese)

[7] Changqing Gao, Shanmin Mi, Wei Chen. A thin wall lightweight folding method and folding device: China, 201410134998.4[P] 2014-06-18. 